Copyright

by

Mitchell Alan Davis

2011 
The Thesis Committee for Mitchell Alan Davis Certifies that this is the approved version of the following thesis:

Monte Carlo simulation of fluorescence imaging of microvasculature

APPROVED BY

SUPERVISING COMMITTEE:

John Pearce, Supervisor

Andrew K. Dunn, Co-Supervisor 
Monte Carlo simulation of fluorescence imaging of microvasculature

\author{
by \\ Mitchell Alan Davis, B.S.Biomed.E.
}

THESIS

Presented to the Faculty of the Graduate School of

The University of Texas at Austin

in Partial Fulfillment

of the Requirements

for the Degree of

MASTER OF SCIENCE IN ENGINEERING

THE UNIVERSITY OF TEXAS AT AUSTIN

August 2011 
Dedicated to my family. 


\section{Acknowledgments}

I would like to thank my advisor, Dr. Andrew Dunn, for all of his assistance and guidance throughout all of this work. I am also grateful to all of my lab-mates for their help and friendship during the course of completing this work. 


\title{
Monte Carlo simulation of fluorescence imaging of microvasculature
}

\author{
Mitchell Alan Davis, M.S.E. \\ The University of Texas at Austin, 2011 \\ Supervisors: John Pearce \\ Andrew K. Dunn
}

Little numerical analysis has been done on fluorescence lifetime imaging in-vivo. Here, a 3D fluorescence Monte Carlo model is used to evaluate a microvasculature geometry obtained via two-photon microscopy. I found that a bulk-vascularization assumption does not provide an accurate picture of penetration depth of the collected fluorescence signal. Instead the degree of absorption difference between extravascular and intravascular space, as well as the absorption difference between excitation and emission wavelengths must be taken into account to determine the depth distribution. Additionally, I found that using targeted illumination can provide for superior surface vessel sensitivity over wide-field illumination, with small area detection offering an even greater amount of sensitivity to surface vasculature. Depth sensitivity can be enhanced by either increasing the detector area or increasing the illumination area. Finally, it is shown that the excitation wavelength and vessel size can affect intra-vessel sampling distribution, as well as the amount of signal that originates from inside the vessel under targeted illumination conditions. 


\section{Table of Contents}

Acknowledgments $\quad$ v

Abstract vi vi vis

List of Tables $\quad$ ix

List of Figures $\quad x$

Chapter 1. Introduction 1

Chapter 2. Three dimensional fluorescence Monte Carlo model 5

2.1 Model Geometry . . . . . . . . . . . . . . . . . . . . . 5

2.2 Photon simulation . . . . . . . . . . . . . . 6

2.2.1 Photon step size . . . . . . . . . . . . . . . . . 7

2.2.2 Voxel boundary interaction . . . . . . . . . . . . . . . . . . . . . . . .

2.2 .3 Scattering . . . . . . . . . . . . . . . . . . 9 9

2.2.4 Absorption and fluorescent photon emission . . . . . . 10

2.2.5 Photon detection . . . . . . . . . . . . . . . . . . 11

2.3 Postprocessing . . . . . . . . . . . . . . . . . . . . . . 12

$\begin{array}{lll}\text { Chapter 3. } & \text { Model geometry and optical properties } & 14\end{array}$

3.1 Three dimensional two-photon geometry . . . . . . . . . . . . 14

3.1.1 Two photon microscopy . . . . . . . . . . . . . . 14

3.1.2 Geometry . . . . . . . . . . . . . . . . 15

3.1.2.1 Two-photon image acquisition . . . . . . . . 15

3.1.2.2 Geometry creation . . . . . . . . . . 16

3.1.3 Optical properties . . . . . . . . . . . . . . 18

3.2 Homogeneous tissue geometry . . . . . . . . . . . . . . . . . 19

3.3 Single vessel geometry . . . . . . . . . . . . . . . . 20 
Chapter 4. Accuracy of the bulk-vascularized tissue assumption 24

4.1 Simulations . . . . . . . . . . . . . . . . . . . . . 24

4.2 Results and Discussion . . . . . . . . . . . . . . . . . . 25

4.3 Conclusion . . . . . . . . . . . . . . . . . . . . . 27

Chapter 5. Evaluation of illumination and detection schemes 29

5.1 Simulations . . . . . . . . . . . . . . . . . . . . 29

5.2 Results and Discussion . . . . . . . . . . . . . . . 30

5.3 Conclusion . . . . . . . . . . . . . . . . . 37

Chapter 6. Intra-vessel distribution of fluorescence emission 39

6.1 Simulations . . . . . . . . . . . . . . . . . . 39

6.2 Results and Discussion . . . . . . . . . . . . . . . . 40

6.3 Conclusion . . . . . . . . . . . . . . . . . . 42

Chapter 7. Conclusion and future work 43

$\begin{array}{ll}\text { Bibliography } & 46\end{array}$

$\begin{array}{ll}\text { Vita } & 52\end{array}$ 


\section{List of Tables}

3.1 Optical properties for microvasculature geometry . . . . . . . 19

3.2 Optical properties for $1 \%$ homogeneous geometry . . . . . . . 20

3.3 Optical properties for $5 \%$ homogeneous geometry . . . . . . 20

3.4 Optical properties for $10 \%$ homogeneous geometry . . . . . . . 21

3.5 Optical properties for single vessel geometry . . . . . . . . . 22 


\section{List of Figures}

2.1 Flow diagram of 3D Fluorescence Monte Carlo simulation. . . 8

$3.1500 \mu m$ by $500 \mu m$ by $400 \mu m$ microvasculature image stack acquired by two-photon scanning microscopy. . . . . . . . . . .

3.2 Geometry file used for single vessel simulations. The 3D matrix of values is integrated along the y dimension to provide an easier viewing angle. The square at the top-center of the geometry represents the vessel. . . . . . . . . . . . . .

4.1 PDF and CDF of collected signal using homogenous, bulkvascularized tissue assumption and comparison to wide-field illumination with small area detection using (a-b) 415nm, (c-d) $524 \mathrm{~nm}$ and (e-f) $800 \mathrm{~nm}$ excitation. Note that the $\mathrm{x}$-axis in (a-b) is different from $(\mathrm{c}-\mathrm{f}) \ldots \ldots \ldots \ldots . \ldots$

5.1 (a) Depth-dependent $(f(z))$ and (b) depth-integrated $(F(z))$ signal distribution for the targeted illumination and detection scheme; (c) and (d) are $f(z)$ and $F(z)$ using the camera scheme, and (e) and (f) are $f(z)$ and $F(z)$ using the confocal scheme.

$5.23 \mathrm{D}$ rendering of fluorescence signal distribution using wide-field illumination with small area detection for $415 \mathrm{~nm}$ excitation. The geometry is a $400 \mu \mathrm{m}$ deep. . . . . . . . . . . . . .

5.3 3D rendering of fluorescence signal distribution using wide-field illumination with small area detection for $524 \mathrm{~nm}$ excitation. The geometry is a $400 \mu \mathrm{m}$ deep. . . . . . . . . . . . . .

5.4 3D rendering of fluorescence signal distribution using wide-field illumination with small area detection for $524 \mathrm{~nm}$ excitation. The geometry is a $800 \mu \mathrm{m}$ deep. . . . . . . . . . . . .

6.1 $100 \mu \mathrm{m}$ single vessel geometry with (a) $415 \mathrm{~nm}$ targeted illumination and (b) 524nm targeted illumination. (c) Amount of detected signal originating from inside the vessel as a function of vessel diameter. 


\section{Chapter 1}

\section{Introduction}

Fluorescence imaging is a widely used method of measuring functional and structural parameters which cannot be easily imaged using endogenous chromophores. The specific uses of fluorophores and phosphors in-vivo vary widely; a few examples include applications such as molecular imaging, cancer imaging, and functional imaging of hemodynamic properties [16]. Correspondingly, there are a number of delivery methods for fluorophores depending on the application in question.

Several imaging methods require the topical application of fluorophores, and subsequent diffusion through the tissue prior to imaging. An example of this method is voltage sensitive dye (VSD) imaging, which is often used to study neuronal firing by measuring calcium flux into and out of cells [5]. When not considering the genetically expressed protein variants, These methods are inherently limited by both the capability of the fluorophore to diffuse through the tissue, and the need to excite the fluorophore and measure the emission through the highly turbid tissue media. However, as these agents distribute themselves in the tissue via diffusion, predicting the distribution of the agent in the tissue can typically be done through the diffusion equation. 
Intravascular contrast agents do not suffer from the need to diffuse through tissue. However, imaging is more difficult to model mathematically as the fluorophore is no longer distributed in a smooth gradient, as is generally the assumption in VSD imaging. Several analytical methods have been used to model photon migration in an intravascular fluorescence imaging context. All of these methods treat the tissue as a homogeneous medium, or a series of homogeneous layers, where diffusion determines the depth and resolution of imaging $[2,9,23]$. In the brain, these methods fail to consider the wide variance in absorption between the intra- and extra-vascular space.

The purpose of this work is to analyze two common intravascular contrast agents used to measure hemodynamic parameters in the brain: porphyrin and Indocyanine Green (ICG). Porphyrin-based contrast agents are oxygen sensitive phosphorescent probes which have excitation maximums at 415 and $524 \mathrm{~nm}$, and an emission maximum at $690 \mathrm{~nm}$. ICG is a fluorescent probe used to image blood flow and clearance, and has an absorption maximum in plasma at approximately $800 \mathrm{~nm}$, and an emission maximum at $880 \mathrm{~nm}$. The dyes were chosen so that several situations could be studied: visible wavelength excitation with NIR emission, and NIR excitation with NIR emission.

Optical in-vivo $\mathrm{pO}_{2}$ measurements using oxygen-sensitive contrast agents have recently become a popular method for evaluating physiological and disease models $[1,8,11,13,22]$. Optical imaging of $p O_{2}$ in-vivo has the advantage of having both high spatial resolution and high temporal resolution [31].

Fluorescence and phosphorescence imaging $[18,22,26]$ in-vivo can be 
broadly classified based on the method of excitation: single photon methods $[1,8,15,20,23,25,27,31]$ rely on diffusion of light into tissue to generate probe emission, while two photon methods allow for depth-selected excitation at a point inside the tissue $[6,19]$. Single photon imaging can be further classified into methods that use targeted excitation $[15,31]$, and methods that use widefield illumination [23].

Several methods have been developed to ensure that excitation is localized, including using scanning confocal microscopy [31] and a Digital Micromirror Device (DMD)[15]. The confocal system also confines the photons detected to a small area in the tissue, while the DMD system integrates the photons emitted from a large area on the tissue surface.

A model has been developed whereby fluorescence and phosphorescence emission can be modeled in a realistic in-vivo geometry using excitation and detection schemes currently being used $[15,24,30]$. The model is used to determine the differences in phosphorescence emission distribution between a realistic microvasculature geometry and bulk tissue geometry. The model is further used to demonstrate the intra-vascular distribution of phosphorescence emission based on the excitation wavelength. The in-vivo geometry is acquired through the use of two-photon microscopy. The theory behind two-photon microscopy, as well as the properties which make it especially suited to providing tissue geometry files for Monte Carlo simulation, are presented later in this work.

The model uses a 3D fluorescence Monte Carlo method to evaluate 
an illumination and detection scheme in an arbitrary geometry[10,12]. Similar non-fluorescence based techniques [14] have been used to study port-wine stain in an epidermal geometry obtained via optical coherence tomography[3]. Geometries are specified by setting tissue optical properties in a 3D voxelized grid. Optical properties for each voxel can be specified for both the excitation light and the emitted light. The modeled excitation photons scatters through the tissue and can either be absorbed in a voxel, whereby an emission event may occur, or terminated if it reaches the boundary of the geometry. Emission photons originate from the location of absorption and are generated moving at random angles. The emission photons can then either scatter or be absorbed based on an independent set of emission optical properties. The theory and details of the Monte Carlo model are presented in detail in the next chapter. 


\section{Chapter 2}

\section{Three dimensional fluorescence Monte Carlo model}

The Monte Carlo model used in this work is a 3D voxelized grid method with fluorescence simulation capabilities. The code was originally adapted from Monte Carlo Multiple Layer (MCML) code, which was developed by Wang and Jaques in 1995 [28] and written in ANSI standard C. The Monte Carlo model simulates the random propagation of photons through an arbitrary geometry with arbirtary optical properties by sampling the step size, scattering, and absorption through the tissue by sampling the appropriate distributions. When a sufficient number of these photons have been simulated, the result is a convergent distribution of photon propagation through tissue. This chapter explains the geometries that the 3D Monte Carlo model is able to use, as well as how photons are propagated through the geometry.

\section{$2.1 \quad$ Model Geometry}

Our 3D Monte Carlo adaptation uses a 3D voxelized grid where the tissue optical properties can be set independently in each voxel. This allows for a 3D variable resolution model of tissue that can be used to emulate true tissue 
geometries. In the scope of this work, a 3D stack of two-photon fluorescence microscopy images were processed and converted to a geometry usable with the fluorescence Monte Carlo model.

Specifically, the geometry is stored in memory as a 3D array of characters, each of which is 8 bits. Each character is a number from 0 to 255, which specifies the tissue type contained in the voxel corresponding to the location in the array. Optical properties are specified by the user in the input file to the program, so when the simulation needs to look up the properties, it uses the tissue type number in the geometry matrix to look up the optical properties.

Several parameters are specified in the optical properties for each voxel. First, the absorption, scattering and anisotropy is specified for the photons entering the geometry. Another set of optical properties are also set for each voxel, which adds the capability to model fluorescence emission. For the emission optical properties, the scattering and absorption is set based on the wavelength of the fluorescence emission, as well as the quantum efficiency (QE). The QE determines the propability of a fluorescence emission event given an absorption in each voxel. In areas where there are no fluorescent molecules, the QE value is set to 0 to represent the lack of fluorescing capabilities in that region.

\subsection{Photon simulation}

The 3D Monte Carlo algorithm is outlined in Fig 2.1. First, a photon is generated at the top of the geometry at a user specified place. The location 
at which the photon enters the tissue is determined by a specified numerical aperture and the focal distance from the top of the geometry. The beam radius is calculated from those parameters, and an entrance point is randomly generated either according to a gaussian or flat, "top hat", distribution around the center of the illumination region. The angle at which the photon enters the tissue is determined by a line drawn from the focal point to the entrance point, and is stored as the cosine of the angle in the $\mathrm{x}, \mathrm{y}$, and $\mathrm{z}$ directions.

\subsubsection{Photon step size}

The intialized photon is then moved in the direction specified by the directional cosines on a number of mean free paths, s, into the tissue. The distance the photon travels into the tissue is referred to as the step size, and is sampled from an exponential distribution according to Beer's law, as seen in Eq. 2.1, where $\mu_{t}$ represents the total attenuation coefficient, which is the sum of the absorption, $\mu_{a}$, and scattering, $m u_{s}$, coefficients in the tissue.

$$
P\left(s \geq s_{1}\right)=e^{-\mu_{t} s_{1}}
$$

To generate a step size, first a uniform random number over $[0,1], \xi$, is sampled using a psuedo random number generator. Eq. 2.2 is then used to convert from a uniform distribution given by the psuedo random number generator to an exponential distribution governed by Beer's law. 


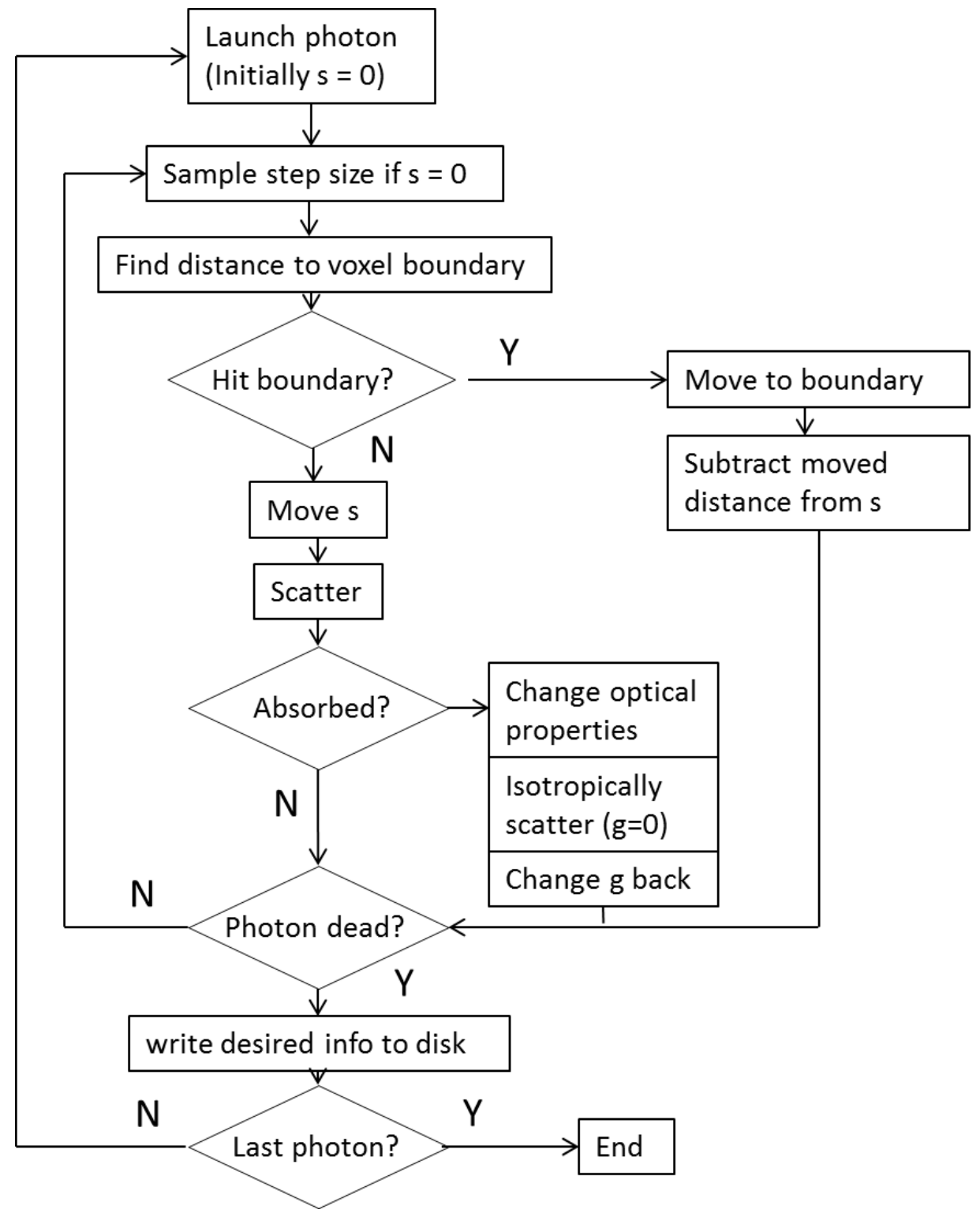

Figure 2.1: Flow diagram of 3D Fluorescence Monte Carlo simulation. 


$$
s=-\frac{\ln (\xi)}{\mu_{t}}
$$

\subsubsection{Voxel boundary interaction}

Once s has been determined, the distance to the nearest voxel boundary is calculated. If the distance to the boundary is less than the distance the photon travels, the photon is moved to the boundary, and the distance moved is subtracted from s. This is done so that if a different tissue type is present in the adjacent voxel, the remaining step size can be recalculated based on the optical properties in the new voxel. Once the remaining s has been calculated for the new voxel, the distance to the next boundary is calculated and compared to the step size remaining. If the photon will hit the boundary, it is moved there as before and $\mathrm{s}$ is recalculated. If it does not hit a boundary, the photon is moved the remainder of the distance s to it's new location.

\subsubsection{Scattering}

When the photon arrives at the new location specified by s, scattering and absorption events occur. Scattering is calculated in both the $\theta$ and $\phi$ directions in the spherical coordinate system originating at the photon location. In the $\theta$ direction, the scattering angle is determined by the Henyey-Green scattering function, Eq. 2.3. The value, g, represents the anisotropy of the scattering, a value near 1 represents almost completely forward scattering, -1 represents backward scattering, and 0 represents isotropic scattering. In tissue, 
for example, the anisotropy value is generally close to 1 . In the $\phi$ direction, the photon has an equal probability of scattering in all directions.

$$
p(\cos \theta)=\frac{1-g^{2}}{2\left(1-g^{2}-2 g \cos \theta\right)}
$$

Two uniformly sampled random numbers, $\xi_{1}$ and $\xi_{2}$, are used to determine the $\theta$ and $\phi$ directions of the scattering in each event. Eq. 2.4 represents the conversion from the uniform distribution given by $\xi_{1}$ to the distribution governed by the Henyey-Greenstein scattering function. Eq. 2.5 represents the transform from a uniform distribution over the range $[0,1]$ to the range $[0,2 \pi]$, which generates the uniformly sampled scattering angle in the $\phi$ direction.

$$
\cos \theta= \begin{cases}\frac{1}{2 g}\left\{1+g^{2}-\left[\frac{1-g^{2}}{1-g-2 g \xi_{1}}\right]\right\} & \text { if } g \neq 0 \\ 2 \xi_{1}-1 & \text { if } g=0\end{cases}
$$

$$
\phi=2 \pi \xi_{2}
$$

\subsubsection{Absorption and fluorescent photon emission}

In this model absorption is initially treated as an all or nothing event. If a photon is absorbed in the tissue, then there is a probability, the quantum efficiency (QE), that an emission photon will be generated. When this happens, the optical properties of the tissue geometry change to the optical properties of the tissue at the emission wavelength. The effect of an emitted 
photon is created by changing the anisotropy value, g, from Eqn 2.3, to 0. This represents an isotropic scattering angle--which properly simulates the isotropic emission from a fluorescent molecule. Once the isotropic scattering angle has been calculated, $\mathrm{g}$ is set to the value that corresponds to the tissue properties at the emission wavelength.

When the fluorescent photon is generated, the Monte Carlo simulation also stops using $\mu_{t}$ to calculate the photon step size. Instead, the $\mu_{t}$ in Eq. 2.2 is replaced by the scattering coefficient, $\mu_{s}$. The advantage of doing this is that the absorption can be evaluated later in the post processing step. In many cases, the scattering of a tissue volume does not change greatly as the wavelength is changed. If the absorption can be done in post-processing, then just one initial simulation can be used to generate results for a number of different emission wavelengths, thereby saving a great deal of computational time. This method has been exploited previously to increase the computational efficiency of solving inverse problems using Monte Carlo simulations [10,21].

\subsubsection{Photon detection}

Initially, the user also sets a center location, radius, and numerical aperture of the area where the photons are detected. Photons which exit the tissue at the top of the geometry, and fall within the guidelines specified by the detector, are flagged to be stored. Photons which exit the tissue outside the detector area are killed and not stored. Upon detection, the entire photon history, including the positions, scattering angles, the distance traveled through 
each tissue type, and origin of the fluorescence absorption event, are parsed. As it is not feasible for limited storage reasons to store all of the movement of every photon in the simulation, the user specifies a subset of the history data to be stored for postprocessing.

\section{$2.3 \quad$ Postprocessing}

In the postprocessing phase, the Beer's law absorption of the emitted photon must be taken into account. While absorption of the incident excitation photons is treated as an all-or-nothing event, the emission photons must be weighted based on the distance they travel through each tissue in the geometry. The contribution to the detected signal from fluorescent photons originating at a point inside the tissue $(\mathrm{x}, \mathrm{y}, \mathrm{z})$ is calculated as:

$$
I_{d}(x, y, z)=\sum_{j} e^{-\sum_{i} \mu_{a}^{i} l_{i j}}
$$

Where $\mathrm{j}$ represents the photons originating at point $(\mathrm{x}, \mathrm{y}, \mathrm{z})$ in the tissue, and i represents the tissue type inside the geometry. The distance each photon travels within each tissue type, $l_{i j}$, is used, along with the absorption coefficient $\mu_{a}^{i}$ in each tissue type, to generate the weight of each photon by Beer's law. This process is repeated for every point inside the geometry to build up a three dimensional distribution of signal using photons that arrive at a user specified detector location.

The depth-dependent, $f(z)$, and depth-integrated, $F(z)$ signal distri- 
butions, representing the depth profile of the fluorescence signals can also be calculated. $f(z)$, which is the probability of the collected signal originating from a depth $z$ in the geometry, is calculated from the signal intensity distribution $I_{d}(x, y, z)$ using the following relation:

$$
f(z)=\frac{\iint I_{d}(x, y, z) d x d y}{\iiint I_{d}(x, y, z) d x d y d z}
$$

$F(z)$ can then be calculated by integrating $f(z)$ from 0 to $z$, as in Eq. 2.8 and represents the amount of signal originating from the first $\mathrm{z} \mu \mathrm{m}$ in the geometry.

$$
F(z)=\int_{0}^{z} f(z) d z
$$




\section{Chapter 3}

\section{Model geometry and optical properties}

In this chapter, the geometry used in all the simulations performed in this work, as well as the optical properties of the tissue at the wavelengths of interest, are given.

The optical properties of porphyrin and ICG were used in the model. The Monte Carlo model allowed for an arbitrary 3D voxelized geometry, where each voxel could represent one of up to 255 different sets of optical properties $\left(\mu_{a}, \mu_{s}^{\prime}, \mathrm{g}\right)$. Quantum efficiency can be set for the emission optical properties, which determines the likelihood of phosphorescence emission upon absorption of an incident excitation photon.

\subsection{Three dimensional two-photon geometry}

\subsubsection{Two photon microscopy}

Two-photon fluorescence microscopy utilizes a long-wavelength excitation photon to generate spatially localized fluorescence. The caveat, however, is that by using the longer wavelength, which in two-photon microscopy is twice as long and has half the energy of the normal excitation wavelength, two photons must be absorbed by a fluorescent molecule nearly simulatenously in 
order to move an electron to a higher energy state. Due to this property, a very high intensity, short pulsed beam must be focused down to a small area. This temporal and spatial concentration of photons increases the probability of a two-photon event occuring, and also inherantly allows for three dimensional imaging. By scanning a focused beam in the $\mathrm{x}, \mathrm{y}$ and $\mathrm{z}$ directions, a 3D mapping of the fluorophores can be obtained.

Though modeling two-photon fluorescence imaging was not my objective, it nevertheless is a useful tool for generating models of tissue that can be used as a geometry in which the photons may propagate in the Monte Carlo simulation.

\subsubsection{Geometry}

\subsubsection{Two-photon image acquisition}

Scanning two-photon microscopy was used to generate depth resolved stacks of microvasculature in a mouse (CD-1; male, $25.30 \mathrm{~g})$. All experimental procedures were approved by the Institutional Animal Care and Use Committee (IACUC) at the University of Texas at Austin. The animals were anesthetized by inhalation of $2.3 \%$ isoflurane in oxygen through a nose cone. Body temperature was maintained at $37^{\circ} \mathrm{C}$ using a feedback controlled heating plate (ATC100, World Percision Instruments, Sarasota, FL, USA) during the experiment. The animals were fixed in a stereotaxic frame (Kopf Instruments, Tujunga, CA, USA) and an $3 \mathrm{~mm} \times 3 \mathrm{~mm}$ portion of the skull was removed using a dental burr (IdealTM Micro-Drill, Fine Science tools, Foster City, CA, 
USA). $50 \mu \mathrm{L}$ of $5 \%$ weight/volume Texas Red (Invitrogen, Eugene, OR, USA) was administered through a retro-orbital injection. A $500 \mu \mathrm{m} \times 500 \mu \mathrm{m}$ image with a resolution of $1 \mu \mathrm{m}$ was taken in $2 \mu \mathrm{m}$ z-steps down to $400 \mu \mathrm{m}$. Fig. 3.1 shows the two-photon stack used to generate the geometry.

\subsubsection{Geometry creation}

The geometry for the Monte Carlo model was then created by filtering the images to smooth out heterogeneities in the vasculature. The smoothing procedure was performed in order to remove the noise present in the image due to the two-photon instrumentation.

As the $\mathrm{x}-\mathrm{y}$ resolution was $1 \mu \mathrm{m}$, and each image represented a tissue section $2 \mu \mathrm{m}$ deeper into the tissue; interpolation was performed in order to create equal resolution in the $\mathrm{x}, \mathrm{y}$ and $\mathrm{z}$ directions. A spline interpolation was performed between each image stacks to generate an image representing the tissue corresponding to $1 \mu \mathrm{m}$ depth intervals. Note that these images do not necessarily represent the true tissue, but this was nevertheless necessary as the Monte Carlo simulation requires cubic voxels to be used to create a geometry file, and a smooth interpolation was considered to be better than filling the middle layers with a duplicate of either of the adjacent images.

The images were then thresholded to remove the intensity mapping and create binary images representing "vasculature" or "no vasculature." The binary images were then converted to a geometry file by creating a 3D matrix of the images. Optical properties representing the intravascular and extravascular 


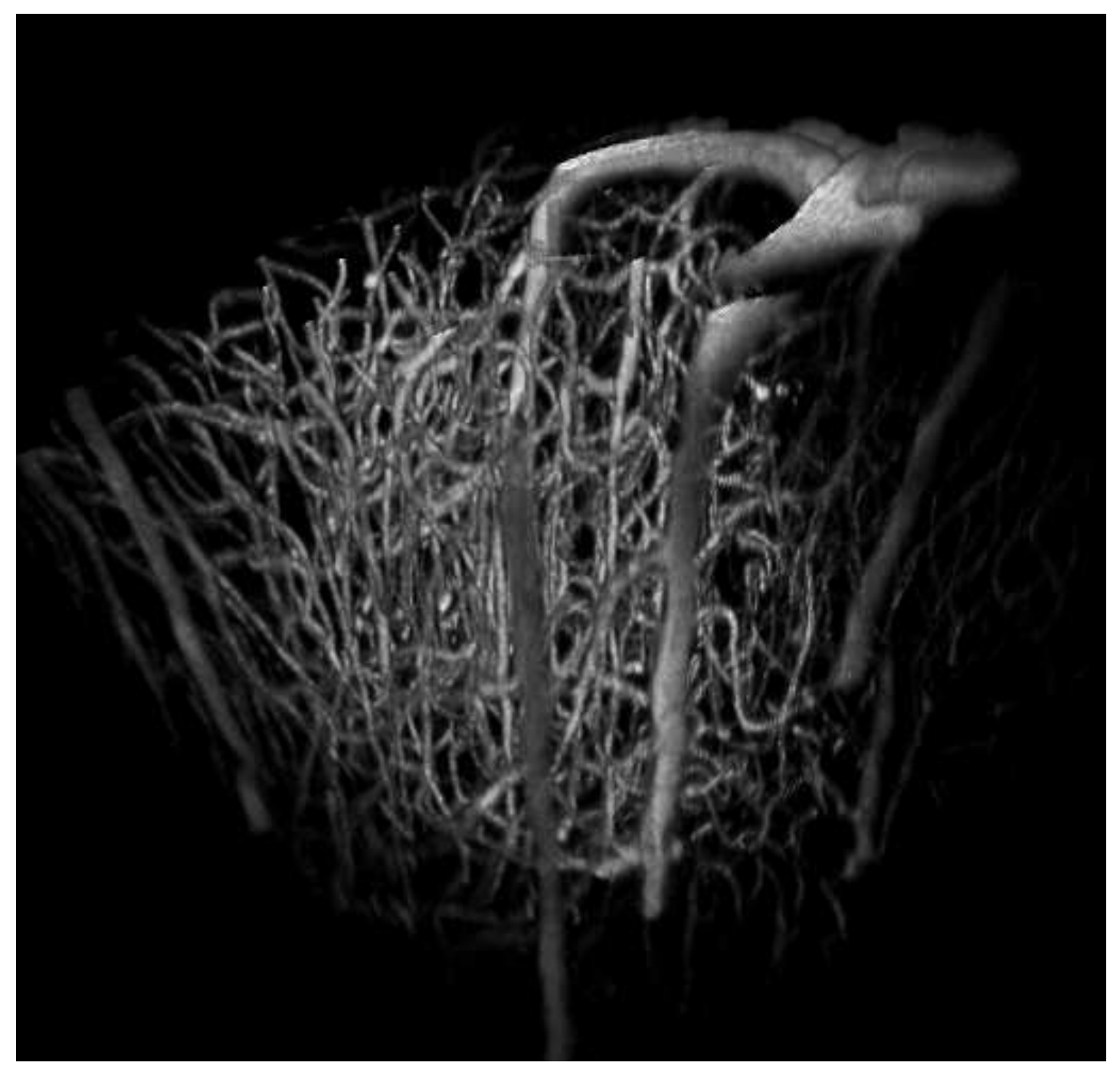

Figure 3.1: $500 \mu \mathrm{m}$ by $500 \mu \mathrm{m}$ by $400 \mu \mathrm{m}$ microvasculature image stack acquired by two-photon scanning microscopy. 
space were assigned to the appropriate sections of the 3D matrix.

\subsubsection{Optical properties}

The intravascular absorption coefficients were generated based on the extinction coefficients of hemoglobin and porphyrin/ICG. Concentration of hemoglobin and porphyrin in the vasculature were assumed to be $150 \mathrm{mg} / \mathrm{ml}[17]$ and $0.3 \mathrm{mg} / \mathrm{ml}[15]$ respectively. The concentration of ICG was $0.1 \mathrm{mg} / \mathrm{ml}$. Scattering coefficients were interpolated from NIR optical property measurements performed by Bevilacqua et al [4] and integrating sphere measurements by Friebel et al[7]. Extravascular absorption and scattering coefficients were based on the in vitro integrating sphere measurements by Yaroslavsky et al[29]. It was necessary to use in vitro measurements of the extravascular tissue because blood was assumed to only be present in intravascular space. The in vitro measurements were taken in the absence of blood. The quantum efficiency was set to 1 for the intravascular space and 0 for the extravascular space. Though realistically the quantum efficiency would be a small fraction of the absorbed photons, the model allowed for $100 \%$ conversion of absorbed excitation photons to emission photons to increase computational efficiency. This simplification is valid because the goal is to predict spatial distribution and relative intensity, not absolute intensity. Table 3.1 shows the optical properties used for the microvasculature geometry. 
Table 3.1: Optical properties for microvasculature geometry

\begin{tabular}{lccc}
\hline & $\mu_{a}\left(\mathrm{~mm}^{-1}\right)$ & $\mu_{s}{ }^{\prime}\left(\mathrm{mm}^{-1}\right)$ & $\mathrm{g}$ \\
\hline Porphyrin & & & \\
\hline 415nm & & 180 & 0.98 \\
\hline Intravascular & 280 & 14 & 0.9 \\
Extravascular & 0.5 & & \\
\hline 524nm & & 120 & 0.98 \\
\hline Intravascular & 16.3 & 11.3 & 0.9 \\
Extravascular & 0.1 & & \\
\hline 690nm & & 100 & 0.98 \\
\hline Intravascular & 0.2 & 10 & 0.9 \\
Extravascular & 0.1 & & \\
\hline ICG & & & \\
\hline 800nm & & 124 & 0.98 \\
\hline Intravascular & 3.75 & 12.4 & 0.9 \\
Extravascular & 0.1 & & \\
\hline 880nm & & 130 & 0.98 \\
\hline Intravascular & 0.7 & 13 & 0.9 \\
Extravascular & 0.1 &
\end{tabular}

\subsection{Homogeneous tissue geometry}

Homogeneous tissue geometries were also created to compare against the realistic geometry. These geometries were created by using a 500x500x400 voxel geometry wherein all voxels were assigned the same properties. Optical properties were assigned by assuming that the only absorber was blood mixed with porphyrin, the same assumption made for the intravascular properties in the microvasculature geometry. The properties were then scaled by $0.01,0.05$ and 0.10 to represent a bulk-vascularization assumption of $1 \%, 5 \%$ and $10 \%$ respectively. The optical properties in detail may be found in Tables 3.2, 3.3 
and 3.4.

Table 3.2: Optical properties for $1 \%$ homogeneous geometry

\begin{tabular}{lccc}
\hline & $\mu_{a}\left(\mathrm{~mm}^{-1}\right)$ & $\mu_{s}{ }^{\prime}\left(\mathrm{mm}^{-1}\right)$ & $\mathrm{g}$ \\
\hline Porphyrin & & & \\
\hline $415 \mathrm{~nm}$ & 2.8 & 14 & 0.9 \\
\hline $524 \mathrm{~nm}$ & 0.163 & 11.3 & 0.9 \\
\hline $690 \mathrm{~nm}$ & 0.001 & 10 & 0.9 \\
\hline$I C G$ & & & \\
\hline $800 \mathrm{~nm}$ & 0.0375 & 12.4 & 0.9 \\
\hline $880 \mathrm{~nm}$ & 0.007 & 13 & 0.9 \\
\hline
\end{tabular}

Table 3.3: Optical properties for 5\% homogeneous geometry

\begin{tabular}{lccc}
\hline & $\mu_{a}\left(\mathrm{~mm}^{-1}\right)$ & $\mu_{s}{ }^{\prime}\left(\mathrm{mm}^{-1}\right)$ & $\mathrm{g}$ \\
\hline Porphyrin & & & \\
\hline $415 \mathrm{~nm}$ & 14 & 14 & 0.9 \\
\hline $524 \mathrm{~nm}$ & 0.815 & 11.3 & 0.9 \\
\hline $690 \mathrm{~nm}$ & 0.01 & 10 & 0.9 \\
\hline$I C G$ & & & \\
\hline $800 \mathrm{~nm}$ & 0.287 & 12.4 & 0.9 \\
\hline $880 \mathrm{~nm}$ & 0.035 & 13 & 0.9
\end{tabular}

\subsection{Single vessel geometry}

Additional geometries were created to determine the effect of targeting a single vessel. A $3 \mathrm{~mm} \times 3 \mathrm{~mm} \times 3 \mathrm{~mm}$ cubic geometry with a single vessel running down the top center was created. The size of the vessel was varied from $25 \mu \mathrm{m}$ to $200 \mu \mathrm{m}$ in $25 \mu \mathrm{m}$ increments. The optical properties were set according to the values found in Table 3.5. Outside the vessel, the tissue was assumed to be homogeneous with a bulk volume fraction of approximately 
Table 3.4: Optical properties for 10\% homogeneous geometry

\begin{tabular}{lccc}
\hline & $\mu_{a}\left(\mathrm{~mm}^{-1}\right)$ & $\mu_{s}{ }^{\prime}\left(\mathrm{mm}^{-1}\right)$ & $\mathrm{g}$ \\
\hline Porphyrin & 28 & 14 & 0.9 \\
\hline $524 \mathrm{~nm}$ & 1.63 & 11.3 & 0.9 \\
\hline $690 \mathrm{~nm}$ & 0.02 & 10 & 0.9 \\
\hline$I C G$ & & & \\
\hline $800 \mathrm{~nm}$ & 0.375 & 12.4 & 0.9 \\
\hline $880 \mathrm{~nm}$ & 0.07 & 13 & 0.9
\end{tabular}

5\%. Fig. 3.2 shows an example single vessel geometry, where the geometry is collapsed along the dimension of the vessel. 
Table 3.5: Optical properties for single vessel geometry

\begin{tabular}{lcccc}
\hline & $\mu_{a}\left(\mathrm{~mm}^{-1}\right)$ & $\mu_{s}^{\prime}\left(\mathrm{mm}^{-1}\right)$ & $\mathrm{g}$ & $\mathrm{QE}$ \\
\hline Porphyrin & \multicolumn{5}{c}{} & & \\
\hline 415nm & & & & \\
\hline Intravascular & 280 & 180 & 0.98 & - \\
Extravascular & 14 & 14 & 0.9 & - \\
\hline 524nm & & & & \\
\hline Intravascular & 16.3 & 120 & 0.98 & - \\
Extravascular & 0.815 & 11.3 & 0.9 & - \\
\hline 690nm & & & & \\
\hline Intravascular & 0.2 & 100 & 0.98 & 1 \\
Extravascular & 0.01 & 10 & 0.9 & 0 \\
\hline ICG & & & & \\
\hline 800nm & & & & \\
\hline Intravascular & 3.75 & 124 & 0.98 & - \\
Extravascular & 0.287 & 12.4 & 0.9 & - \\
\hline 880nm & & & & \\
\hline Intravascular & 0.7 & 130 & 0.98 & 1 \\
Extravascular & 0.035 & 13 & 0.9 & 0 \\
\hline
\end{tabular}




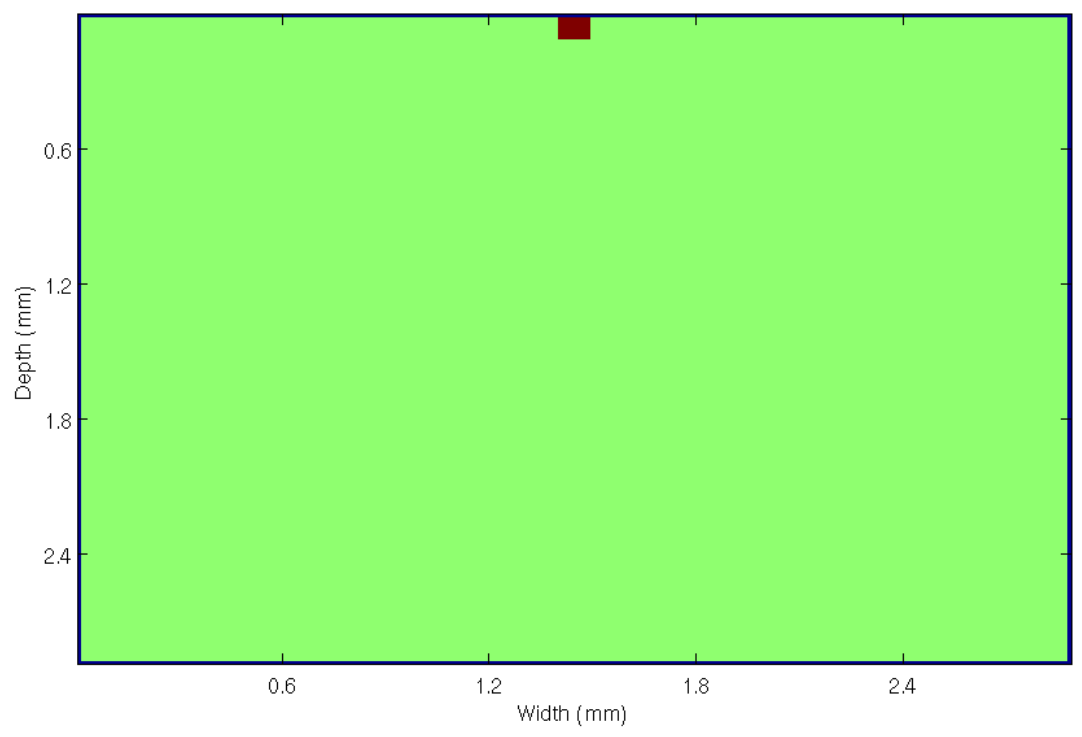

Figure 3.2: Geometry file used for single vessel simulations. The 3D matrix of values is integrated along the y dimension to provide an easier viewing angle. The square at the top-center of the geometry represents the vessel. 


\section{Chapter 4}

\section{Accuracy of the bulk-vascularized tissue assumption}

The first consideration of this work was to analyze the difference in the origin of emission between an in vivo microvasculature geometry, and the more typically used assumption of homogeneous tissue with a volume fraction of absorbers. First, the simulations used to determine the accuracy of the homogeneous tissue assumption will be presented, followed by the results and a discussion of their implications.

\subsection{Simulations}

In the microvasculature geometry, a widefield illumination and small detection method were simulated. A $400 \mu \mathrm{m}$ diameter, slightly diverging (NA $=0.01)$ cone of light was used as the illumination. The detector was a $50 \mu \mathrm{m}$ $\mathrm{x} 50 \mu \mathrm{m}$ square at the top-center of the geometry. Imaging techniques which rely on a full surface illumination and a camera for detection operate on a similar principle. The small detection volume can be thought of as a single pixel in a camera. This illumination and detection scheme will henceforth be referred to as the "camera" scheme. 
For each of the three homogeneous tissue geometries, $1 \%, 5 \%$, and $10 \%$ blood volume fraction, simulations were performed using $415 \mathrm{~nm}$ and $524 \mathrm{~nm}$ excitation wavelengths. As with the microvasculature geometries, a wide-field illumination method with a small area detector was used. $f(z)$ and $F(z)$ were calculated from the signal distribution, $I_{d}(x, y, z)$ using Eqns. 2.7 and 2.8, and used to compare the homogeneous and microvascular geometries.

\subsection{Results and Discussion}

The comparison between the microvasculature geometry and the homogeneous tissue geometry can be seen in Fig. 4.1. The left column of Fig. 4.1 shows the depth-dependent signal distribution, $f(z)$, of the collected signal distribution, calculated using Eq. 2.7. The right column of Fig. 4.1 shows the depth-integrated signal distribution, $F(z)$, of the collected signal as a function of depth-a value of 0.3 at $100 \mu \mathrm{m}$, therefore, would mean that $30 \%$ of the fluorescence signal originated from the top $100 \mu \mathrm{m}$ of the geometry.

For reference, measurements of the volume of the microvasculature geometry showed that the approximate volume fraction of blood vessels was $3.5 \%$. Homogenous geometries of $1 \%, 5 \%$, and $10 \%$ for $415 \mathrm{~nm}$ and $524 \mathrm{~nm}$ excitation and $5 \%$ and $10 \%$ for $800 \mathrm{~nm}$ excitation are shown to demonstrate that the results do not match a realistic geometry in most cases. The results suggest that the accuracy of a bulk-vascularized tissue assumption is strongly dependent on the excitation wavelength of the fluorophore.

The 415nm excitation case shown in Fig. 4.1(a) shows that the homo- 
genenous tissue assumption significantly underestimates the depth from which the collected signal originates. Even a 1\% vascularized assumption shows $90 \%$ of the signal originating from the first $100 \mu \mathrm{m}$, whereas the simulation shows that $90 \%$ of the signal comes from the first $250 \mu \mathrm{m}$. Differences in signal distribution can be attributed to the large difference between the intra and extra vascular absorption coefficients. Fig. 5.1(a) shows that a significant portion of hte signal fromes from surface vasculature over the illumination area and the first vessel below the detector, at $40 \mu \mathrm{m}$ below the tissue. Excitation photons experience a small amount of absorption outside of the blood vessels, which allows them to scatter around blood vessels and penetrate deeper into the tissue. The emission photons, being Stokes-shifted into the near infrared region, are not strongly absorbed in any tissue and can return to the surface without much attenuation from absorption.

$524 \mathrm{~nm}$ and $800 \mathrm{~nm}$ excitation produced very different results. Fig. 4.1(c-d) shows that with $524 \mathrm{~nm}$ excitation the microvasculature geometry simulation gives a depth-dependent signal distribution less than that of even a $10 \%$ vacularized homogeneous tissue geometry. While the $524 \mathrm{~nm}$ excitation case still shows shallower penetration than would be expected given the $3.5 \%$ vascularized geometry used, the error is less dramatic than the $800 \mathrm{~nm}$ case. 800nm excitation with 880nm emission, shown in Fig. 4.1(e-f) again produced the opposite result from the $415 \mathrm{~nm}$ excitation, where the depth penetration is significantly less than what would be expected. $1 \%$ vacularization was not shown, as $90 \%$ of the signal originated from the first $7-8 \mathrm{~cm}$, which is far deeper 
than the approximately $400 \mu \mathrm{m}$ depth penetration exhibited by the photons in the microvasculature geometry.

Based on the results, it appears that the depth penetration in a realistic geometry relative to the homogenous geometry assumption strongly correlates with the amount of difference between the absorption of blood and contrast agent at the excitation wavelength and the emission wavelength. A large excitation-emission absorption difference, such as the $415 \mathrm{~nm}$ to $690 \mathrm{~nm}$ in porphyrin, causes the depth of penetration to be greater than what the homogeneous assumption would predict. A smaller difference, on the other hand, appeared to produce the opposite effect, with the homogeneous tissue assumption significantly overpredicting the depth of the collected signal distribution.

\subsection{Conclusion}

Here, I show that using a homogeneous geometry assumption to approximate the depth-distribution of the collected fluorescence signal is not an accurate method. A more complex model, involving both the difference in blood absorption at the excitation and emission wavelengths, as well as the difference in absorption coefficients in the fluorescing region compared to the non-fluorescing region is required to provide a more accurate picture of depth penetration. 


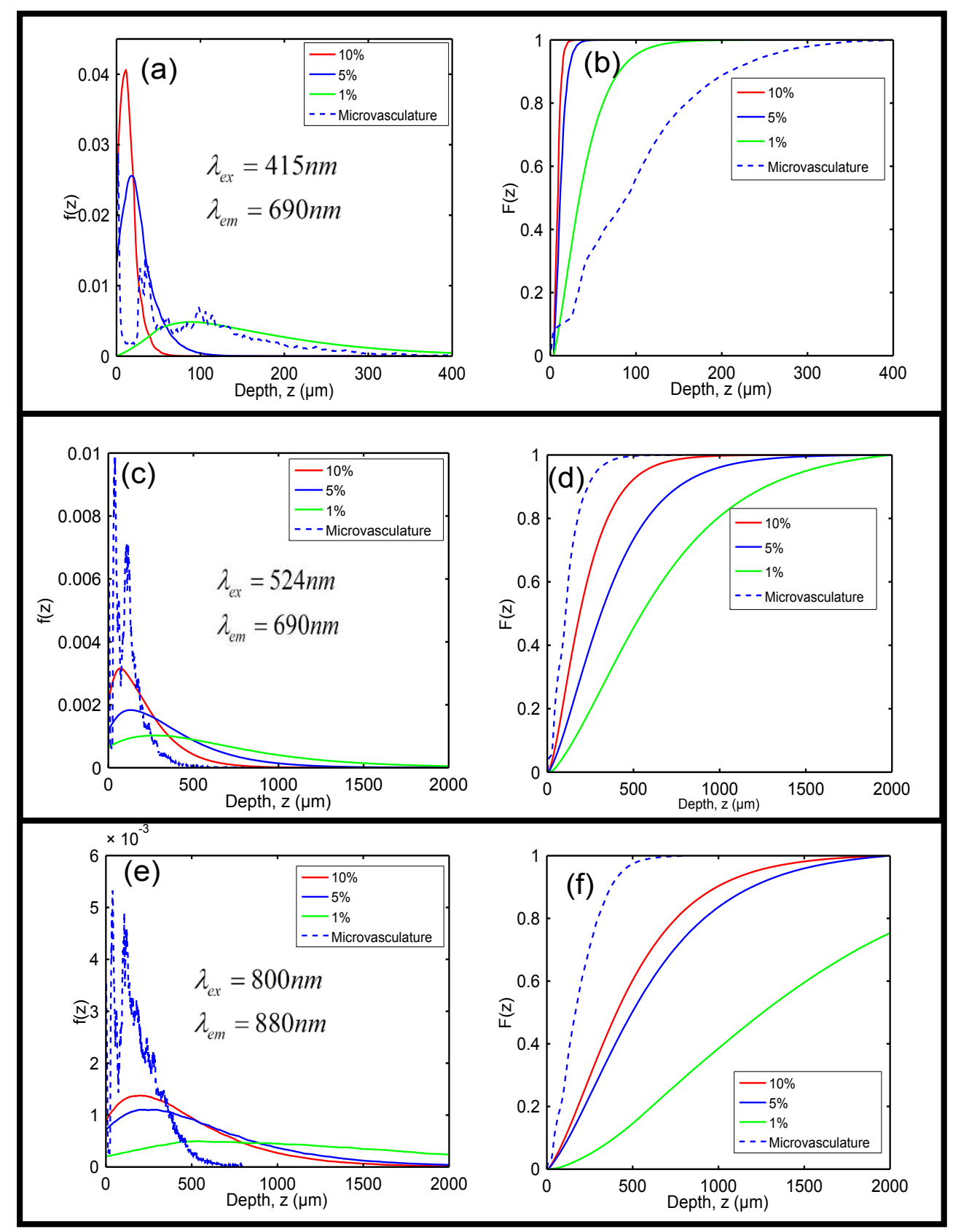

Figure 4.1: PDF and CDF of collected signal using homogenous, bulkvascularized tissue assumption and comparison to wide-field illumination with small area detection using (a-b) 415nm, (c-d) 524nm and (e-f) 800nm excitation. Note that the $\mathrm{x}$-axis in $(\mathrm{a}-\mathrm{b})$ is different from $(\mathrm{c}-\mathrm{f})$. 


\section{Chapter 5}

\section{Evaluation of illumination and detection schemes}

\subsection{Simulations}

For each excitation wavelength $(415 \mathrm{~nm}, 524 \mathrm{~nm}$ and $800 \mathrm{~nm})$ the microvascular geometry was used for three illumination and detection schemes. The "camera" illumination and detection scheme described previously was used for the comparison, in addition to two other methods referred to as the "targeted" method and the "confocal" method.

The second method consisted of a $20 \mu \mathrm{m}$ diameter illumination area and a detector which captured all emission photons exiting the surface of the tissue. This scheme mimics selective illumination of a small area of the tissue and integration of all fluorescent photons exiting the tissue, similar to approaches that utilize structured excitation with single point detectors[15]. This scheme will be referred to as the "targeted" illumination and detection scheme.

The third method was a hybrid of the previous two, which is similar to the scanning confocal method. The illumination source was a $20 \mu \mathrm{m}$ diameter beam with an NA of 0.01 , while the detector was a $50 \mu \mathrm{m}$ x $50 \mu \mathrm{m}$ square at the 
top-center of the geometry. This method is similar to the results provided when the laser scanning confocal method[31] is used, and as such will be referred to as the "confocal" scheme.

The results of the three illumination schemes were then compared to determine the effect of choosing a targeted illumination scheme over a wide-field illumination scheme, as well as a wide field detector (i.e. PMT or APD) over a point detector (i.e. camera pixel) based on the desired signal distribution.

To provide a comparison between the schemes, both the depth-dependent and depth-integrated signal distributions for each excitation and emission wavelength pair were calculated according to Eqns. 2.7 and 2.8. Additionally, the $3 \mathrm{D}$ rendering of the signal distribution for the camera scheme, $I_{d}(x, y, z)$, was generated to demonstrate the effects of using a widefield illumination method.

\subsection{Results and Discussion}

Figure 5.1 shows the depth dependence of the detected signals for the three imaging geometries. Fig. 5.1(a) shows the depth-dependent signal distribution, $f(z)$, in the microvasculature geometry using the camera illumination and detection scheme at 415nm, 524nm and 800nm excitation. Fig. 5.1(c) shows $f(z)$ when using a wide-field illumination method with a small detection area on the surface of the tissue, and Fig. 5.1(e) shows $f(z)$ in the case of the confocal illumination and detection scheme. Figs. 5.1(b), 5.1(d) and 5.1(f), on the right column, show the depth-integrated signal distribution, 
$\mathrm{F}(\mathrm{z})$, at each depth in the tissue under the illumination and detection scheme corresponding to Figs. 5.1(a), 5.1(c) and 5.1(e).

The depth-dependent signal distribution plots represent the probability of the collected fluorescent photons originating from each depth in the tissue. From Fig. 5.1(a), there is approximately $0 \%$ probability of photons originating from $100 \mu \mathrm{m}$ into the tissue, while there is a $0.5 \%$ chance of photons originating from $100 \mu \mathrm{m}$ into the tissue in the $1 \%$ and microvasculature geometries. The depth-integrated plots, on the other hand, represent the total amount of fluorescence originating from above a depth, $\mathrm{z} \mu \mathrm{m}$, in the tissue. In Fig. 5.1(b), for example, $50 \%$ of collected fluorescence originates from the first $5-10 \mu \mathrm{m}$ in the $5 \%$ and $10 \%$ homogeneous tissues, about $25 \mu \mathrm{m}$ in the $1 \%$ homogeneneous tissue, and the first $100 \mu \mathrm{m}$ in the realistic in vivo microvasculature geometry.

Given the results, it is clear that the best spatial localization is obtained when using the confocal illumination and detection scheme. The strong peak at approximately $40 \mu \mathrm{m}$ represents an approximately $20 \mu \mathrm{m}$ wide vessel directly below the surface position of the illumination beam. This shows that given a low numerical aperture (NA) beam, surface vasculature can be strongly emphasized by localizing both the illumination and the detection positions.

Using targeted or point illumination with a wide-area detector generates a strongly wavelength-dependent signal distribution. When using $415 \mathrm{~nm}$ excitation, the signal distribution is similar to the distribution obtained when using the confocal scheme. However, when $524 \mathrm{~nm}$ or $800 \mathrm{~nm}$ excitation light is used, the first vessel encountered did not represent a significant fraction 


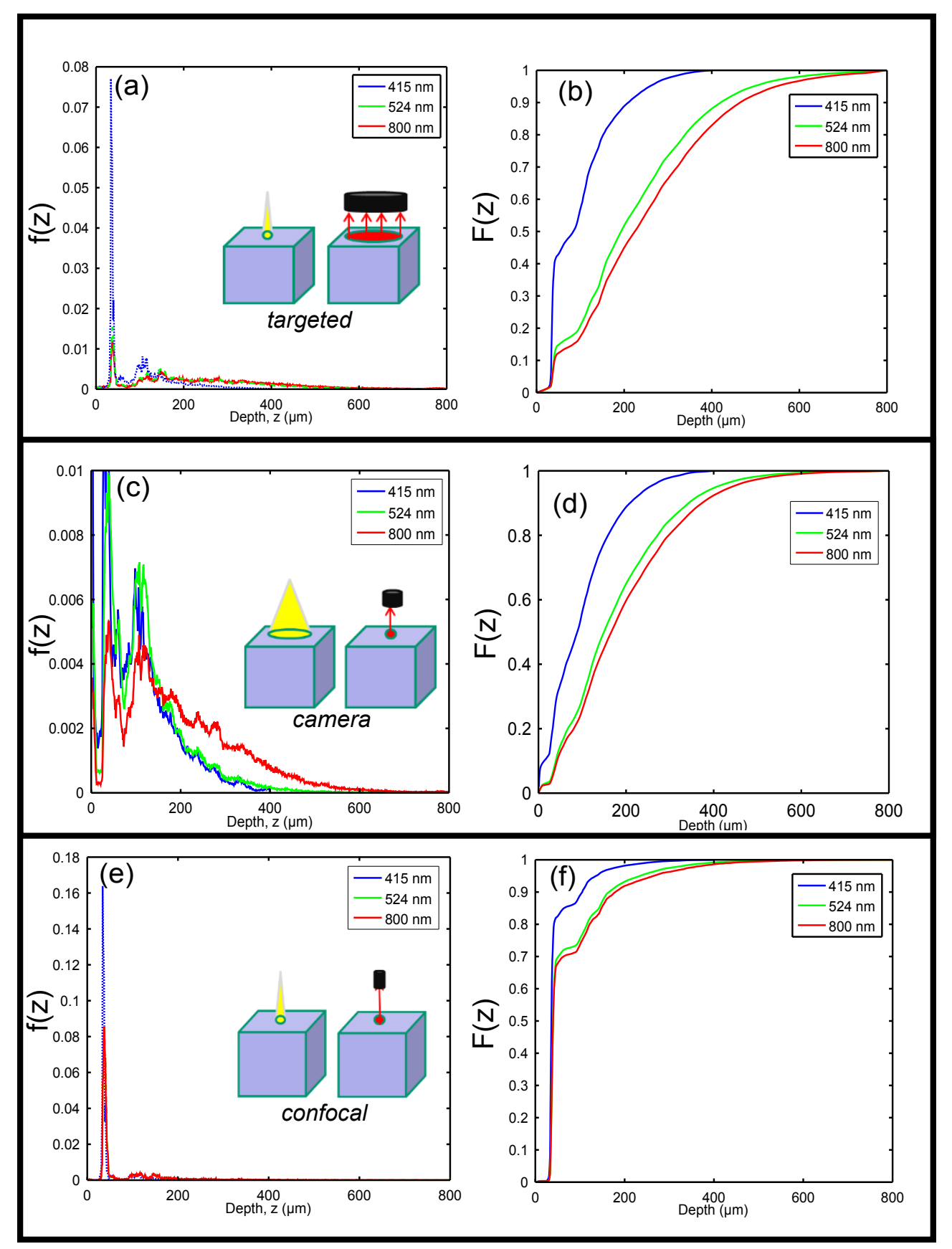

Figure 5.1: (a) Depth-dependent $(f(z))$ and (b) depth-integrated $(F(z))$ signal distribution for the targeted illumination and detection scheme; (c) and (d) are $f(z)$ and $F(z)$ using the camera scheme, and (e) and (f) are $f(z)$ and $F(z)$ using the confocal scheme. 
of the total measured signal. This observation could be useful in selecting a wavelength for excitation, depending on the goal or target of imaging. With porphyrin, for example, either the surface vasculature can be emphasized by using $415 \mathrm{~nm}$ excitation light, or higher depth sensitivity can be obtained by using $524 \mathrm{~nm}$ excitation light.

Lastly, the camera scheme shown in Fig. 5.1(c-d), is the least sensitive method of the three to surface vasculature, with only a small fraction of the total collected signal originating from directly under the detector area. Regardless of the excitation wavelength used, the light entering the detector is not representative of the surface vasculature below the detector area. This is an important observation, as often the surface vasculature will be visible on the camera which is detecting the fluorescence, and it is easy to mistake the signal received as coming from the surface vessels. However, even in the case of the $415 \mathrm{~nm}$ excitation light, less than $10 \%$ of the detected signal originated from the vessel below the detector area.

Figs. 5.2, 5.3 and 5.4 show 3D renderings of the origin of detected fluorescence generated using the camera illumination and detection scheme, as generated by weighting each detected photon according to Eq. 2.6. The total signal was then normalized to generate a 3D PDF of the signal using $1 \mu \mathrm{m} \times 1 \mu \mathrm{m}$ bins. The simuation demonstrates that when a large area on the surface is illuminated, the fluorescence signal collected at the surface can originate not only from deeper in the tissue under the detector area, but also from areas near the surface that are not under the detector: similar to what 


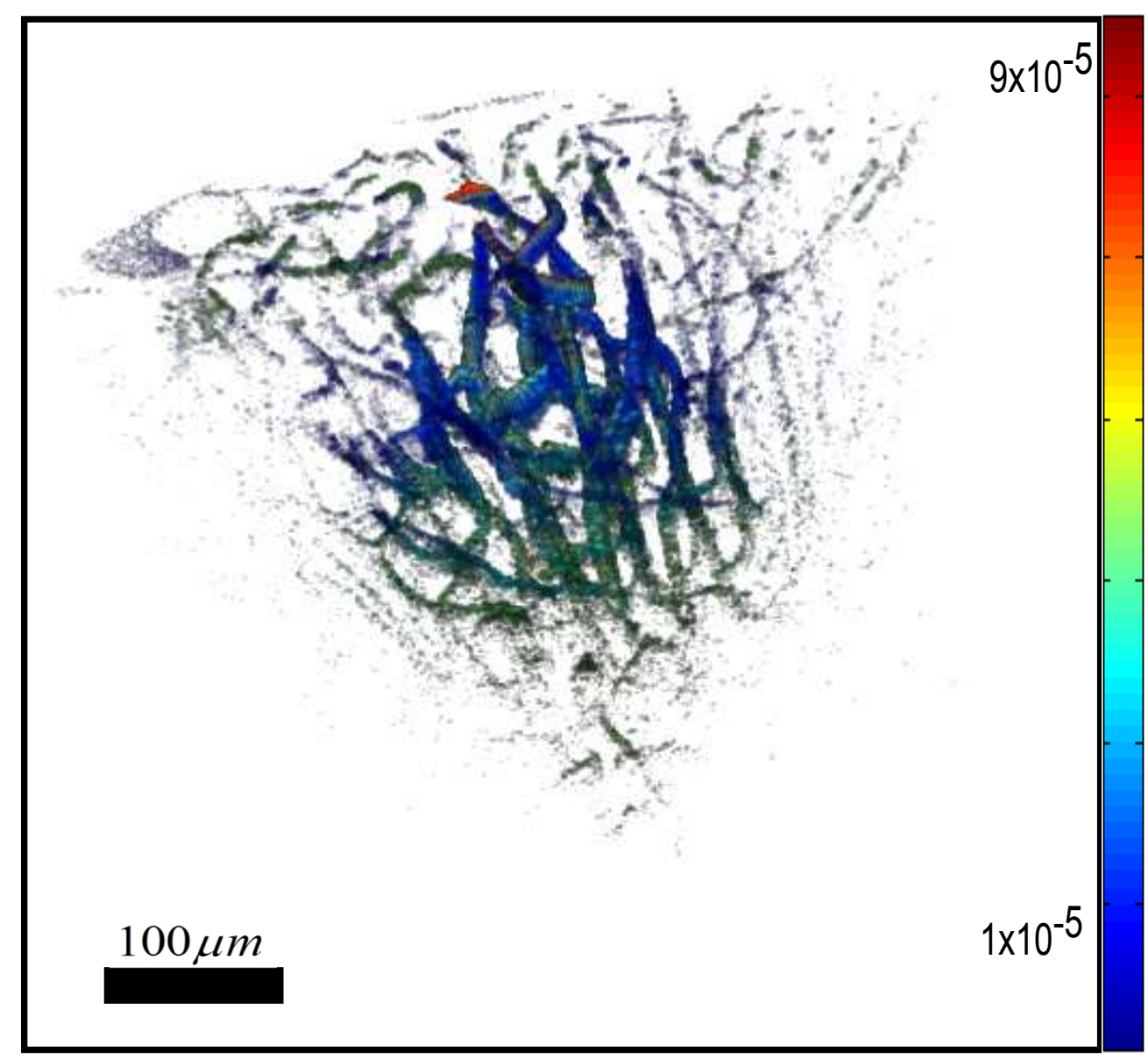

Figure 5.2: 3D rendering of fluorescence signal distribution using wide-field illumination with small area detection for $415 \mathrm{~nm}$ excitation. The geometry is a $400 \mu \mathrm{m}$ deep. 


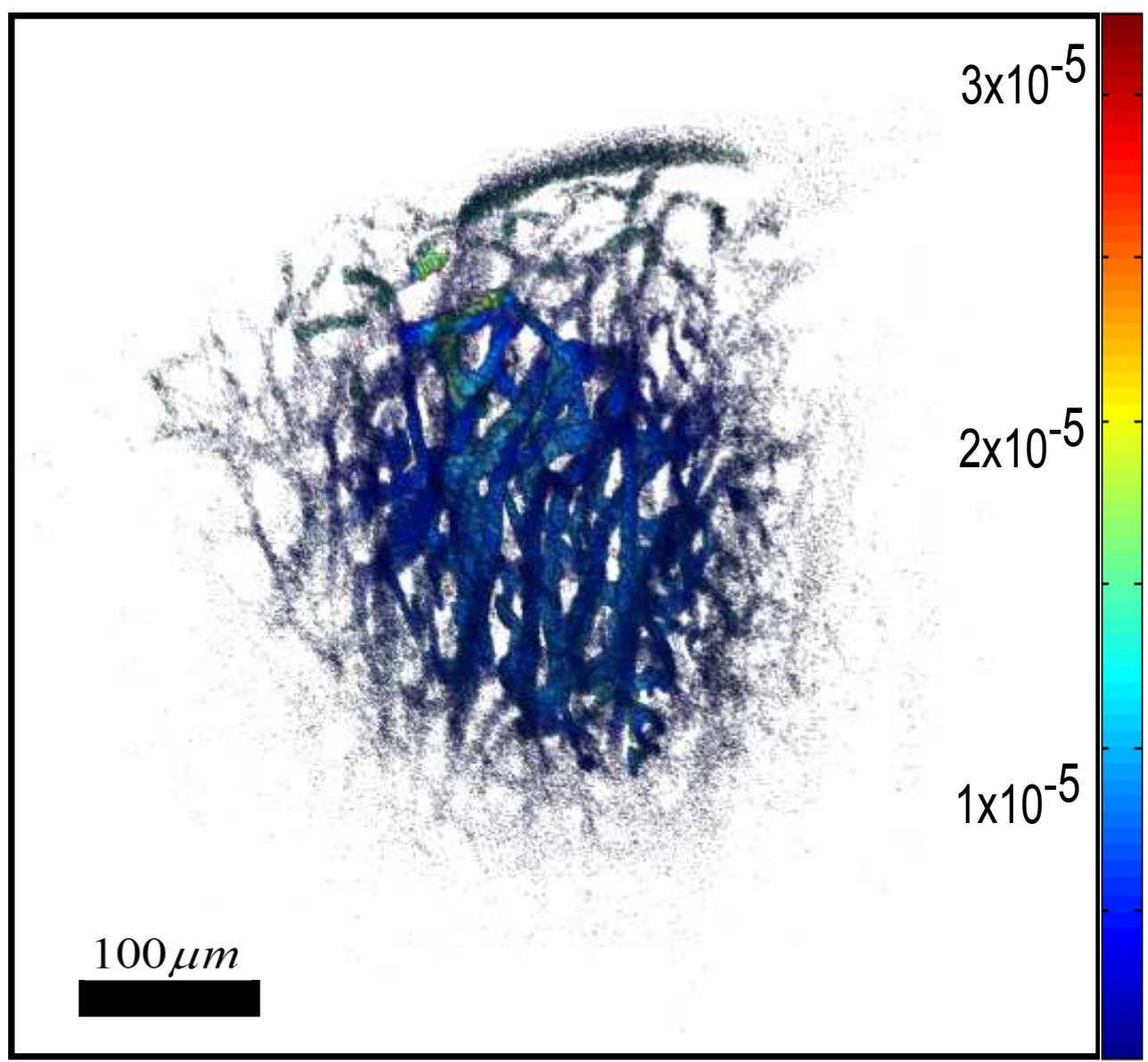

Figure 5.3: 3D rendering of fluorescence signal distribution using wide-field illumination with small area detection for $524 \mathrm{~nm}$ excitation. The geometry is a $400 \mu \mathrm{m}$ deep. 


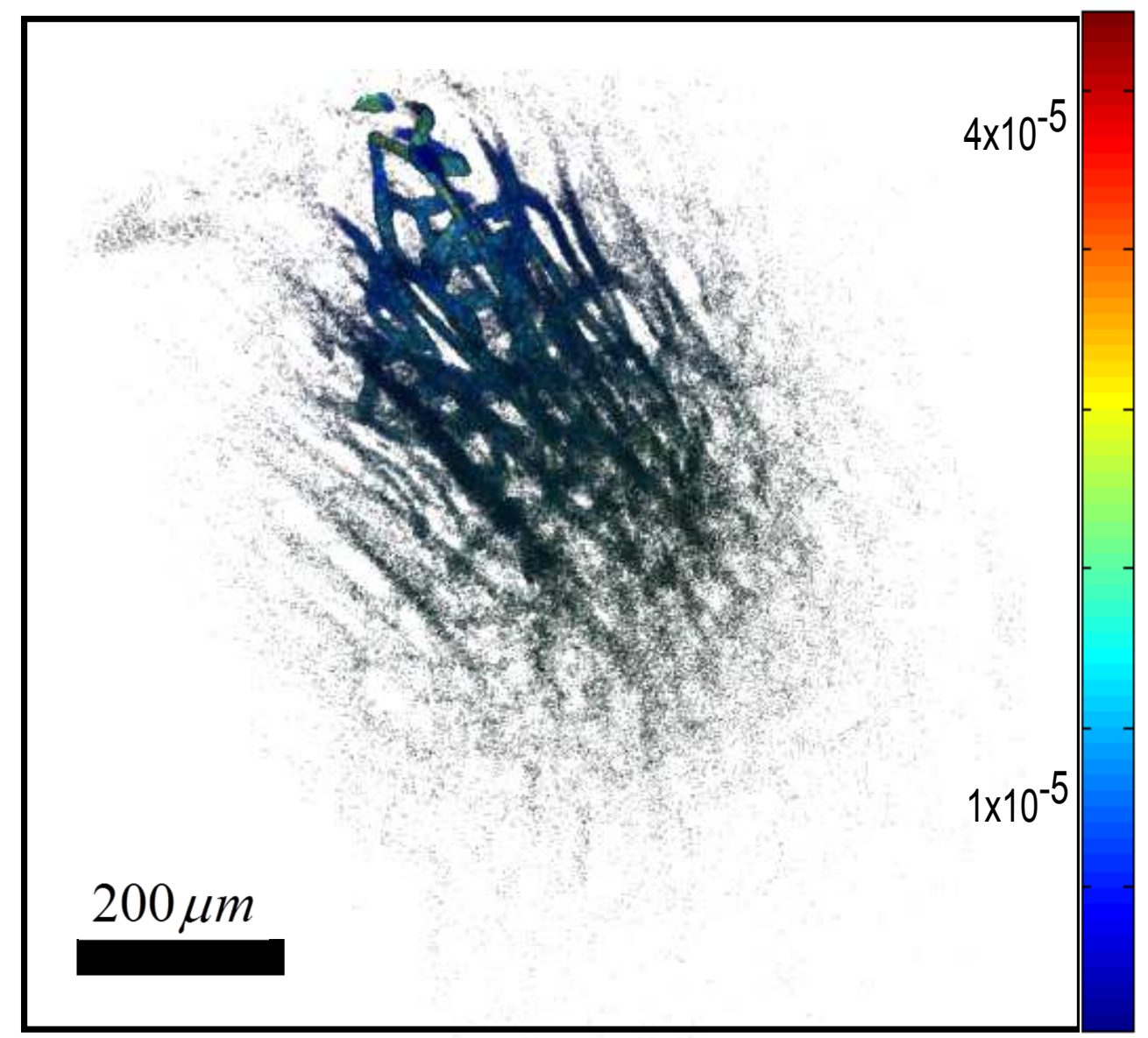

Figure 5.4: 3D rendering of fluorescence signal distribution using wide-field illumination with small area detection for $524 \mathrm{~nm}$ excitation. The geometry is a $800 \mu \mathrm{m}$ deep. 
is seen with source-detector separations. The renderings also provide a good visual aid to explain the peaks in Fig. 5.1(a), 5.1(c) and 5.1(e). The peaks at the surface correspond to the signal detected from the surface vasculature under the entire illumination area, while the peaks at $40 \mu \mathrm{m}$ correspond to the first vessel directly beneth the detector surface.

The results suggest that techniques that prioritize accurate imaging of microvasculature should aim to focus the excitation light on the tissue of interest. If the excitation light has a small NA (nearly collimated), then the emitted light will represent vasculature at a number of different depths, dependent on the distance between the surface and the first vessel the excitation light meets. In Fig. 5.1(f), for example, the excitation photons scatter down to $40 \mu \mathrm{m}$ before encountering a blood vessel. Techniques that utilize depth selection independently of illumination would benefit from taking this observation into consideration.

\subsection{Conclusion}

Three illumination and detection schemes used in fluorescence imaging have been evaluated to determine the effect of imaging technique on the origin of the collected signal. Targeted illumination schemes offer a more focused signal distribution over wide field illumination. Using a camera with targeted illumination in particular offers very strong localization of signal to the surface vasculature, while wide-area detection can provide more depth-weighted signals. Wide-area illumination with small area detection offers the greatest 
depth weighting of the three illumination and detection schemes that were evaluated, but at the cost of including fluorescence from near the surface over the entire illumination region. 


\section{Chapter 6}

\section{Intra-vessel distribution of fluorescence emission}

The purpose of modeling fluorescence emission from a single vessel is primarily to provide a more accurate picture regarding what happens when a vessel is targeted with excitation light. The primary question is: if a surface vessel is targeted, will the fluorescence signal returning from the tissue be representative of that vessel? Though this is similar to the simulations done in the previous chapter, with the targeted illumination schemes, here I look at vessels that are on the surface of the tissue. Examples where this is the case in the brain would be the dural vessels, which lie along the top of the surface of the cerebrum.

\subsection{Simulations}

The objective of the single vessel geometry was to determine the penetration of excitation light into underlying tissue, and thus to determine how much of the captured signal can be attributed to the targeted vessel. As such, the illumination area was a circle with a diameter that matched the diameter of the vessel in each geometry. Vessel diameters of 25, 50, 75, 100, 150, 
and $200 \mu \mathrm{m}$ were used. Simulations were performed using $415 \mathrm{~nm}, 524 \mathrm{~nm}$ and $800 \mathrm{~nm}$ illumination and a wide-field detector. Outside of the vessel, a 5\% volume fraction of vascularization was used so that a measure of the amount of excitation light penetrating through the vessel could be easily measured.

\subsection{Results and Discussion}

Fig. 6.1 shows the results of single vessel geometry simulations at $524 \mathrm{~nm}$ and 415nm illumination. Figs. 6.1(a) and 6.1(b) show a simulation result when the vessel size was set to $100 \mu \mathrm{m}$. The 3D distribution of the signal, $I_{d}(x, y, z)$ was integrated along the length of the vessel to generate the images in Figs. 6.1(a) and 6.1(b). A figure demonstrating single vessel penetration of $800 \mathrm{~nm}$ excitation light is not shown, as it visually appears similar to the 524nm excitation case. Fig. 6.1(c) shows the fraction of the total collected signal which originated inside the $100 \mu \mathrm{m}$ vessel at each of the three excitation wavelengths. A targeted surface vessel with a diameter of $100 \mu \mathrm{m}$, for example, will produce a signal that is $100 \%$ representative of the vessel fluorescence at $415 \mathrm{~nm}, 80 \%$ representative of vessel fluorescence at $524 \mathrm{~nm}$, and $50 \%$ representative of vessel fluorescence at $800 \mathrm{~nm}$.

At $415 \mathrm{~nm}$, seen in Fig. 6.1(c), the intra-vessel absorption was high enough to completely prevent the excitation light from penetrating through to deeper tissues. The absorption also prevented the excitation light from being able to sample the entire vessel. This could effect measurements where an average across the vessel diameter would provide a more accurate measurement 
of a given parameter than just the value near the vessel wall. The $524 \mathrm{~nm}$ illumination light was able to sample the entire vessel, but at smaller vessel sizes the light also penetrated through the vessel and sampled a significant amount of tissue outside the vessel in question. At $800 \mathrm{~nm}$, the results are similar to the $524 \mathrm{~nm}$ excitation case in Fig. 6.1(b), though as expected more light is able to penetrate through the vessel. Note that while these results demonstrate that using 415nm illumination can allow for strong emphasis of surface vasculature in the targeted illumination scheme, Fig. 4.1 also suggests that using this wavelength can result in emphasized surface vasculature in all three of the illumination and detection schemes evaluated.

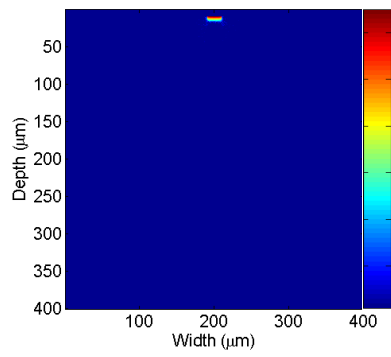

(a)

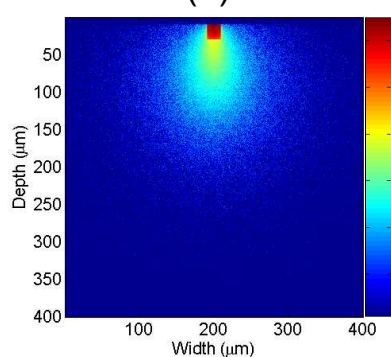

(b)

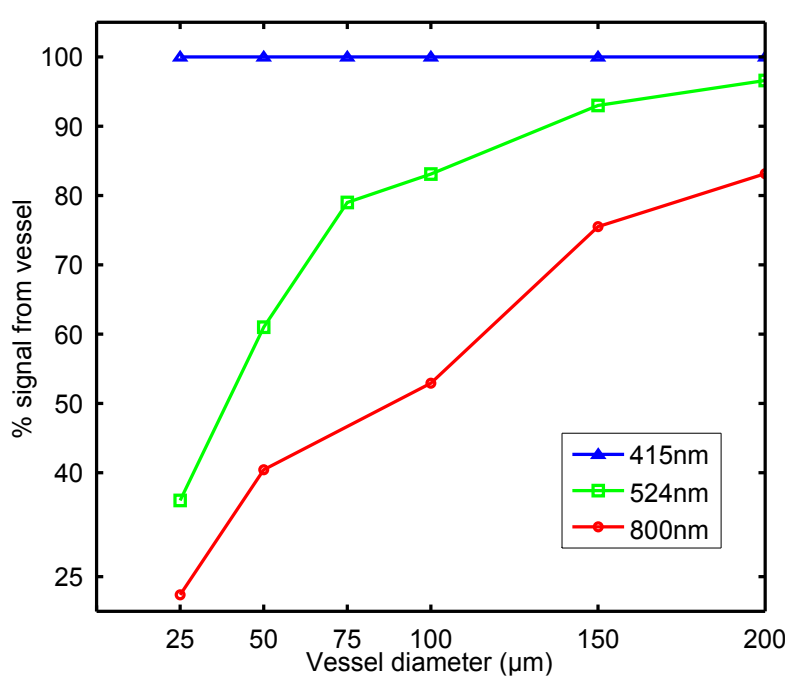

(c)

Figure 6.1: $100 \mu \mathrm{m}$ single vessel geometry with (a) $415 \mathrm{~nm}$ targeted illumination and (b) $524 \mathrm{~nm}$ targeted illumination. (c) Amount of detected signal originating from inside the vessel as a function of vessel diameter. 


\subsection{Conclusion}

Here, I examined intra-vessel sampling and demonstrated that when using an excitation wavelength that is strongly absorbed by blood, the collected signal originates from only the top-surface of the targeted vessels. This may need to be taken into account if using a lifetime imaging method that will produce different results near the vessel walls-a wavelength which is absorbed by blood less may be more useful to provide a signal which is integrated across the vessel diameter. 


\section{Chapter 7}

\section{Conclusion and future work}

In this work, several characteristics of fluorescence lifetime imaging in the brain that have not to my knowledge been previously investigated were studied.

First, it was shown that using a homogeneous geometry assumption to approximate the depth-distribution of the collected fluorescence signal is not an accurate method. A more complex model, involving both the difference in absorption at the excitation and emission wavelenths, as well as the difference in absorption coefficients in the fluorescing region compared to the non-fluorescing region is required to provide a more accurate picture of depth penetration.

Additionally, three illumination and detection schemes used in fluorescence imaging were examined. Targeted illumination schemes offer a more focused signal distribution over wide field illumination. Using a camera with targeted illumination, in particular, offers very strong localization of signal to the surface vasculature, while wide-area detection can provide more depthweighted signals. Wide-area illumination with small area detection offers the greatest depth-weighting of the three illumination and detection schemes that 
were evaluated, but at the cost of including fluorescence from near-the-surface over the entire illumination region.

Lastly, I examined intra-vessel sampling and demonstrated that when using an excitation wavelength that is strongly absorbed by blood, the collected signal originates from only the top-surface of the targeted vessels. This may need to be taken into account if using a lifetime imaging method that will produce different results near the vessel walls-a wavelength which is absorbed by blood less may be more useful to provide a signal which is integrated across the vessel diameter.

It is my hope that these results will aid design considerations when building or purchasing an instrument to do intravascular fluorescence imaging.

In the future, I would like to perform a more intensive study of the fluorescence imaging dynamics by using arbitrary excitation and emission wavelengths. If the absorption properties of the constrast agent may be disregarded, as is often the case in blood since it is such a strong absorber, then the optical properties across the entire visible spectrum may be easily calculated. The difficulty, however, lies with the computational time required to run that many simulations. One simulation would need to be run for each excitation wavelength desired, though the emission wavelength would be changable through postprocessing. As an example, to simulate the 800nm excitation case using the camera scheme, over 10 billion photons were used to generate a convergent fluorescence distribution. This simulation took approximately 6 days of compute time to run. 
As computational time is the biggest limiter of a larger, more intensive study of this kind, the next step would be to parallelize the fluorescence Monte Carlo code. I plan to do this by implementing the algorithm in CUDA C, which by moving the computation to a graphics processing unit (GPU), has the potential to speed up the simulation run times several orders of magnitude. 


\section{Bibliography}

[1] B M Ances, D F Wilson, J H Greenberg, and J a Detre. Dynamic changes in cerebral blood flow, $\mathrm{O} 2$ tension, and calculated cerebral metabolic rate of O2 during functional activation using oxygen phosphorescence quenching. Journal of cerebral blood flow and metabolism : official journal of the International Society of Cerebral Blood Flow and Metabolism, 21(5):5116, May 2001.

[2] S R Arridge, M Cope, and D T Delpy. The theoretical basis for the determination of optical pathlengths in tissue: temporal and frequency analysis. Physics in medicine and biology, 37(7):1531-60, July 1992.

[3] Jennifer Barton, T. Pfefer, Ashley Welch, Derek Smithies, Jerry Nelson, and Martin Van Gemert. Optical Monte Carlo modeling of a true portwine stain anatomy. Optics Express, 2(9):391, April 1998.

[4] Frederic Bevilacqua, Dominique Piguet, Pierre Marquet, Jeffrey D Gross, Bruce J Tromberg, and Christian Depeursinge. In vivo local determination of tissue optical properties : applications to human brain. Applied Optics, 38(22), 1999.

[5] Lawrence B. Cohen and Brian M. Salzberg. Optical Measurement of Membrane Potential. Rev. physiol. Biochem. Pharmacol., 83:36-77, 
January 1978.

[6] Arnold D Estrada, Adrien Ponticorvo, Tim N Ford, and Andrew K Dunn. Microvascular oxygen quantification using two-photon microscopy. $O p$ tics letters, 33(10):1038-40, May 2008.

[7] Moritz Friebel, Klaus Do, Andreas Hahn, Gerhard Mu, D Berlin, Laser Medizin, and Freie Universita. Optical properties of circulating human blood in the wavelength range 400 - $2500 \mathrm{~nm}$. Journal of biomedical optics, 4(1):36-46, 1999.

[8] Aleksander S Golub and Roland N Pittman. PO2 measurements in the microcirculation using phosphorescence quenching microscopy at high magnification. American journal of physiology. Heart and circulatory physiology, 294(6):H2905-16, June 2008.

[9] D Hattery, V Chernomordik, M Loew, I Gannot, and a Gandjbakhche. Analytical solutions for time-resolved fluorescence lifetime imaging in a turbid medium such as tissue. Journal of the Optical Society of America. A, Optics, image science, and vision, 18(7):1523-30, July 2001.

[10] C K Hayakawa, J Spanier, F Bevilacqua, A K Dunn, J S You, B J Tromberg, and V Venugopalan. Perturbation Monte Carlo methods to solve inverse photon migration problems in heterogeneous tissues. Optics letters, 26(17):1335-7, September 2001. 
[11] G. Helmlinger, F. Yuan, M. Dellian, and R.K. Jain. Interstitial pH and pO2 gradients in solid tumors in vivo: high-resolution measurements reveal a lack of correlation. Nature Medicine, 3(2):177-182, 1997.

[12] A.T. Kumar, J Skoch, B.J. Bacskai, D.A. Boas, and A.K. Dunn. Fluorescencelifetime-based tomography for turbid media. Optics letters, 30(24):33473349, 2005.

[13] Egbert G Mik, Tanja Johannes, and Can Ince. Monitoring of renal venous PO2 and kidney oxygen consumption in rats by a near-infrared phosphorescence lifetime technique. American journal of physiology. Renal physiology, 294(3):F676-81, March 2008.

[14] T.J. Pfefer, J. Kehlet Barton, E.K. Chan, M.G. Ducros, B.S. Sorg, T.E. Milner, J.S. Nelson, and a.J. Welch. A three-dimensional modular adaptable grid numerical model for light propagation during laser irradiation of skin tissue. IEEE Journal of Selected Topics in Quantum Electronics, 2(4):934-942, 1996.

[15] Adrien Ponticorvo and Andrew K Dunn. Simultaneous imaging of oxygen tension and blood flow in animals using a digital micromirror device. Optics express, 18(8):8160-70, April 2010.

[16] Jianghong Rao, Anca Dragulescu-Andrasi, and Hequan Yao. Fluorescence imaging in vivo: recent advances. Current opinion in biotechnology, 18(1):17-25, February 2007. 
[17] Francisco E Robles, Shwetadwip Chowdhury, and Adam Wax. Assessing hemoglobin concentration using spectroscopic optical coherence tomography for feasibility of tissue diagnostics. Optics Express, 1(1):310-317, 2010.

[18] W.L. Rumsey, J.M. Vanderkooi, and D.F. Wilson. Imaging of phosphorescence: a novel method for measuring oxygen distribution in perfused tissue. Science, 241(4873):1649, 1988.

[19] Sava Sakadžić, Emmanuel Roussakis, Mohammad a Yaseen, Emiri T Mandeville, Vivek J Srinivasan, Ken Arai, Svetlana Ruvinskaya, Anna Devor, Eng H Lo, Sergei a Vinogradov, and David a Boas. Two-photon highresolution measurement of partial pressure of oxygen in cerebral vasculature and tissue. Nature methods, 7(9):755-759, August 2010.

[20] Sava Sakadzić, Shuai Yuan, Ergin Dilekoz, Svetlana Ruvinskaya, Sergei A Vinogradov, Cenk Ayata, and David A Boas. Simultaneous imaging of cerebral partial pressure of oxygen and blood flow during functional activation and cortical spreading depression. Applied Optics, 48(10):D16977, April 2009.

[21] Angelo Sassaroli, Costantino Blumetti, Ffbrizio Martelli, Lucia Alianelli, Daniele Contini, Andrea Ismaelli, and Giovanni Zaccanti. Monte carlo procedure for investigating light propagation and imaging of highly scattering media. Applied optics, 37(31):7392-400, November 1998. 
[22] R.D. Shonat and P.C. Johnson. Oxygen tension gradients and heterogeneity in venous microcirculation: a phosphorescence quenching study. American Journal of Physiology- Heart and Circulatory Physiology, 272(5):H2233H2240, 1997.

[23] Vadim Soloviev, David Wilson, and Sergei Vinogradov. Phosphorescence lifetime imaging in turbid media: the forward problem. Applied Optics, 42(1):113-23, January 2003.

[24] Vadim Y Soloviev, David F Wilson, and Sergei a Vinogradov. Phosphorescence lifetime imaging in turbid media: the inverse problem and experimental image reconstruction. Applied Optics, 43(3):564-74, January 2004.

[25] IP Torres Filho and M Intaglietta. Microvessel PO2 measurements by phosphorescence decay method. American Journal of Physiology- Heart and Circulatory Physiology, 265(4):H1434, 1993.

[26] J M Vanderkooi, G Maniara, T J Green, and D F Wilson. An optical method for measurement of dioxygen concentration based upon quenching of phosphorescence. The Journal of biological chemistry, 262(12):547682, April 1987.

[27] Sergei A Vinogradov, Maria A Fernandez-searra, Benjamin W Dugan, and David F Wilson. Frequency domain instrument for measuring phosphorescence lifetime distributions in heterogeneous samples. Review of Scientific Instruments, 72(8):3396-3406, 2001. 
[28] L Wang, S L Jacques, and L Zheng. MCML-Monte Carlo modeling of light transport in multi-layered tissues. Computer methods and programs in biomedicine, 47(2):131-46, July 1995.

[29] A N Yaroslavsky, P C Schulze, I V Yaroslavsky, R Schober, F Ulrich, and H J Schwarzmaier. Optical properties of selected native and coagulated human brain tissues in vitro in the visible and near infrared spectral range. Physics in medicine and biology, 47(12):2059-73, June 2002.

[30] Mohammad A Yaseen, Vivek J Srinivasan, Sava Sakadžić, Harsha Radhakrishnan, Iwona Gorczynska, Weicheng Wu, James G Fujimoto, and David A Boas. Microvascular oxygen tension and flow measurements in rodent cerebral cortex during baseline conditions and functional activation. Journal of cerebral blood flow and metabolism, pages 1-13, December 2010.

[31] Mohammad A Yaseen, Vivek J Srinivasan, Sava Sakadzić, Weicheng Wu, Svetlana Ruvinskaya, Sergei a Vinogradov, and David a Boas. Optical monitoring of oxygen tension in cortical microvessels with confocal microscopy. Optics express, 17(25):22341-50, December 2009. 


\section{Vita}

Mitchell Davis was born December 5, 1985 in Houston, TX. He received his bachelor's degree in Biomedical Engineering from the University of Texas at Austin in May of 2009. After completing his degree, he enrolled in the University of Texas at Austin for his graduate work in Electrical Engineering. Currently, he is pursuing his PhD in Electrical Engineering and hopes to finish by 2014 .

Permanent address: 13118 Campos Dr.

Houston, Texas 77065

This thesis was typeset with $\mathrm{AT}_{\mathrm{E}} \mathrm{X}^{\dagger}$ by the author.

\footnotetext{
${ }^{\dagger}$ LTEX is a document preparation system developed by Leslie Lamport as a special version of Donald Knuth's TEX Program.
} 